\title{
Uncovering the hidden meaning of cross-curriculum comparison results on the Force Concept Inventory
}

\author{
Lin Ding \\ ${ }^{1}$ Department of Teaching and Learning, The Ohio State University, Columbus, Ohio 43210, USA \\ Marcos D. Caballero \\ ${ }^{2}$ Department of Physics and Astronomy and the CREATE for STEM Institute, \\ Michigan State University, East Lansing, Michigan 48824, USA
}

(Received 22 May 2014; published 6 November 2014)

\begin{abstract}
In a recent study, Caballero and colleagues conducted a large-scale evaluation using the Force Concept Inventory (FCI) to compare student learning outcomes between two introductory physics curricula: the Matter and Interactions (M\&I) mechanics course and a pedagogically-reformedtraditional-content (PRTC) mechanics course. Using a conventional approach to detect betweencurriculum differences, the researchers found that students in the PRTC course outperformed their M\&I peers on nearly all the FCI items. However, this conventional approach does not control for the levels of student mastery of the tested concepts, and hence falls short of revealing deep information about the key differences between the two curricula. To this end, we use a stratum-specific method, common for detecting differential item functioning, to analyze a subset of the empirical data in the study of Caballero et al. By comparing students of equal mastery levels measured by the FCI (stratumspecific analysis), we find that the M\&I students in fact demonstrate an advantage on the questions regarding Newton's first and second laws. In addition, the previously reported underperformance of the M\&I students on kinematics diminishes in our stratum-specific analysis. Results from this study show better alignment with students' exposure to relevant topics in their respective courses. Both our findings and methodological approach provide useful implications for research on effective curriculum evaluation.
\end{abstract}

DOI: 10.1103/PhysRevSTPER.10.020125

PACS numbers: 01.40.Fk, 01.40.G-, 01.40.gf, 01.40.Di

\section{INTRODUCTION}

The physics education research (PER) community has been a pioneer on the frontier of curriculum reform for the past few decades [1,2]. Many efforts geared toward transforming introductory physics courses have been carried out successfully. These efforts include pedagogical reforms targeting effective strategies of course delivery [3-9], as well as content reforms aimed at reshaping the goals and structure of course content [10-13]. In evaluating the effectiveness of these efforts, comparative studies [14] are often conducted using a common assessment instrument (for example, a concept inventory) with students in both reformed and traditional classes prior to and following instruction [15,16]. Results from such comparisons can reveal a great deal of information regarding the strengths and limitations of new pedagogies and curricula. To this end, assessments and particularly their proper use play a

Published by the American Physical Society under the terms of the Creative Commons Attribution 3.0 License. Further distribution of this work must maintain attribution to the author(s) and the published article's title, journal citation, and DOI. critical role for drawing valid educational inferences that can inform evidence-based course transformation.

Valid assessments, from a broad perspective, can be used to assist learning, monitor student progress, and evaluate the efficacy of educational programs. From the more specific perspective of curriculum development, assessments can directly or indirectly impact researchers' decision making and hence can have significant ramifications on the course and future directions of reform endeavors. It is recognized that valid inferences of educational outcomes require not only carefully designed assessments but also proper measurement models for accurate analysis and interpretation $[17,18]$. In light of comparative studies, this means that student performance on a chosen assessment sometimes needs to be examined at a fine-grained level, beyond primary between-group comparisons, in order to reveal otherwise hidden information regarding the nature of different curricula.

Typically, a common practice in many comparative studies is to compare, in aggregate, assessment results between two groups of students. That is, students are not stratified into different levels of mastery or proficiency for matched analysis as discussed by Clauser and Mazor [19] but taken as a whole for cross-curriculum comparisons. 
Results based on such comparisons only yield broad-stroke information about student performance in each group, but much of the deep meaning that is informative of student educational exposure is lost. Even if comparisons are made for individual questions, the results are still at a lower order of precision, missing fine-grained evidence necessary to explicate higher-order differences between the curricula under investigation-differences that can only be revealed through stratified comparisons at different levels of mastery intended by the assessment.

For instance, a recent study conducted by Caballero and colleagues [16] used the Force Concept Inventory (FCI) [20] to probe student understanding of Newtonian concepts in both a pedagogically-reformed-traditionalcontent (PRTC) physics course and a reformed course called Matter and Interactions (M\&I) [11]. The researchers compared student performance on the overall FCI and on the individual items between the two courses. By placing the results in the context of what each course emphasizes, Caballero et al. found that students in the M\&I course demonstrated a consistently lower performance not only on the entire assessment but also on nearly all the individual items. While the researchers carefully considered many potential confounders, the comparisons in their study were carried out at the curriculum level; that is, students in each curriculum (PRTC or M\&I) were lumped together for a collective comparison without being divided into different strata to match on their proficiency levels measured by the FCI.

In the present study, we seek to reexamine the empirical data collected in the study of Caballero et al. by using a finer-grained approach common for detecting differential item functioning (DIF). Through this approach, we look beyond the surface meaning of the aggregate results on the FCI by taking groups of students who earned the same total score for matched comparisons. This allows us to uncover deeper information regarding the outcomes of the M\&I and PRTC mechanics courses, which was untapped in the prior study. Specifically, by drawing on the DIF framework, this study is aimed to answer the following research questions:

(1) To what extent do FCI questions exhibit DIF between the students in the M\&I mechanics course and those in the PRTC course?

(2) How do the patterns of detected DIF inform us of the cross-curriculum similarities and differences?

In what follows, we first introduce M\&I Modern Mechanics in contrast to traditional introductory mechanics courses. We then lay a theoretical foundation for DIF and its connection to the varying learning opportunities of students in different curricula. A brief description of the methods used in the study along with background information is then introduced, followed by detailed analysis and findings. Based on the results, discussions of possible inferences about the M\&I and PRTC curricula are provided. In addition, we discuss the implications of the present study for future research on curriculum development and comparative evaluation.

\section{MATTER AND INTERACTIONS MODERN MECHANICS}

Matter and Interactions is a calculus-based physics course that transforms the structure of introductory physics by focusing on fundamental principles, connecting microscopic behavior to macroscopic phenomenon, and making use of numerical computation [21]. While many introductory mechanics courses follow a historical canon of topics, M\&I Modern Mechanics is organized around three fundamental principles: (1) the momentum principle — external forces cause changes to a system's momentum $\left(\Delta \vec{p}_{\text {syst }}=\right.$ $\left.\vec{F}_{\text {ext }} \Delta t\right)$; (2) the energy principle-energy changes in a system are a result of the work done on and heat exchanged with the system's surroundings $\left(\Delta E_{\text {syst }}=W+Q\right)$; and (3) the angular momentum principle-external torques cause changes to a system's angular momentum $\left(\Delta \vec{L}_{s y s t}=\right.$ $\vec{\tau}_{\text {ext }} \Delta t$ ). As these principles are introduced in the above sequence throughout the course, students use them in concert to solve problems. That is, each principle is not treated in the absence of the others that have been previously introduced. Differing from the traditional courses in which the topic of atomic systems only receives a cursory discussion, M\&I Modern Mechanics deliberately connects the microscopic behaviors of atoms to macroscopic phenomena. Students apply the three fundamental principles to atomic systems and engage in principled discussions of contact forces, thermal energy, and entropy $[22,23]$. Additionally, the M\&I course introduces students to modern problem-solving tools such as numerical computation and computer visualization [13]. Early in the course, students learn to iteratively predict motion using the Euler-Cromer method [24] and to write programs that model the motion of various systems using the VPython programming environment [25]. It has been shown that students with no programming experience can successfully model the motion of new and unfamiliar situations [26].

The historical canon of force and motion topics is followed by many introductory physics courses. Most traditional mechanics courses start with kinematics, which is the description of the motion of objects without any discussion of the forces responsible for the motion. The underlying dynamics are later unpacked after students learn Newton's laws, but these discussions are limited to constant force motion in order for problems to be solved analytically. Typically in this portion of the course, a number of different forces are encountered including the frictional force, the normal force, and the tension force. These forces are often presented without a mention of atoms or with only a cursory discussion of the role of 


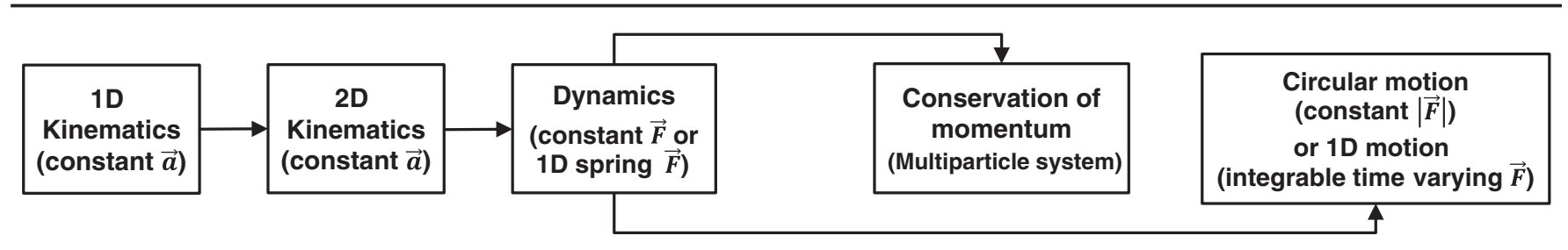

(a)

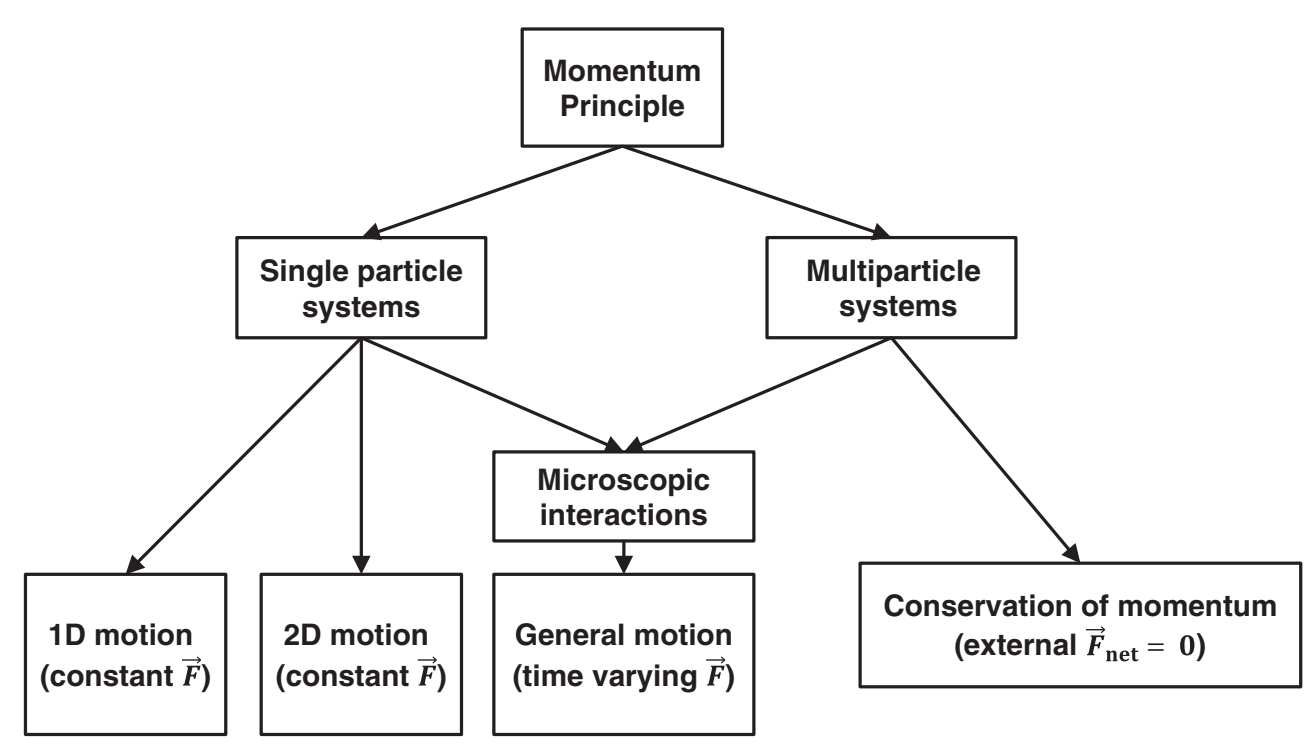

(b)

FIG. 1. The organizing structures of traditional mechanics courses and M\&I modern mechanics. (a) The traditional canon of linearly sequenced force and motion concepts; arrows indicate the topical progression in traditional courses. (b) The hierarchical organization of force and motion concepts in M\&I modern mechanics; arrows indicate the topical encapsulation from an overarching principle to specific topics.

atoms in generating these forces. These courses then switch to the topic of energy conservation that includes the introduction of multiparticle systems. After introducing multiparticle systems, a typical physics course then returns to the discussion of force and motion (in multiparticle systems) through the conservation of linear momentum. The motion of single particles in circular trajectories also follows, but the discussion is limited to situations where the force is of constant magnitude. Occasionally, the order of the last two topics (conservation of linear momentum and circular motion) is flipped. This canon is represented Fig. 1(a).

With regard to force and motion, the M\&I approach leverages a systems-based hierarchy where Newton's first and second laws are presented in the general form of the momentum principle as the governing basis of motion. The course begins with a discussion of single particle systems, but motion in one and two dimensions is treated dynamically. That is, students study how a constant net force changes the momentum of objects, and how the resulting motion can be predicted analytically by leveraging the definition of average velocity. At the same time, students learn to iteratively predict the motion of objects due to a constant force by applying Newton's second law and the definition of average velocity in discrete steps [13,24]. Here, the net force is unpacked as resulting from individual interactions. Contact interactions (friction, the normal force, and tension) are introduced at the microscopic level through the spring-ball model of solids [23]. "Actions at a distance" are later discussed in the context of the gravitational force. At this time, students have developed the computational skills to numerically model the motion of objects interacting through any general force law, which they use to model the motion of a spring-mass oscillator in $3 \mathrm{D}$ and the general motion of planetary bodies. It is precisely through this iterative prediction of motion that the generalizable nature of the momentum principle is uncovered. Newton's laws govern all motion in mechanics, and it is simply the interactions that differ between systems. After this more general discussion of the motion of single particle systems, multiparticle systems are discussed and the conservation laws of energy and linear momentum follow. The conceptual framework for the treatment of force and motion in M\&I mechanics appears in Fig. 1(b). 


\section{FRAMEWORK OF DIFFERENTIAL ITEM FUNCTIONING}

Differential item functioning is a special term coined by the educational measurement community to refer to situations in which an item (question) functions differentially for different groups of students. Ideally, students' probability of answering a given question correctly should be solely determined by their proficiency or mastery levels measured by the test in which the question is included [19,27-29]. So, regardless of their group identity, if students are matched on the target proficiency or mastery level, their odds of answering the question correctly should be more or less the same $[19,27,28]$. This is not to say that students in different groups must perform exactly the same on any question. True differences do exist between student groups as a result of being exposed to different types of instruction or curricula. However, if a difference persists after students are matched on the intended proficiency level, then this difference is considered as DIF, suggesting that what this item is testing with one group of students may be different from what it is testing with the other group [30]. This warrants further inspection and can lead to our better understanding of some higher-order cross-group differences-differences that are otherwise masked in aggregate comparisons.

Two underlying mechanisms can cause DIF: content effect and context effect [31,32]. The content effect is derived primarily from the different instruction or intervention students have received [28]. If a group of students has more access to a particular topic than another group of students, then perceivably the former group is likely to perform better than the latter on this topic. For example, if a test item measures student knowledge about musical instruments, then those who are more frequently exposed to live orchestras are likely to have a higher probability of answering it correctly than those who are not. This content effect, in principle, reflects a divergence in learning opportunities to which students in different groups may have been exposed [30].

The other underlying cause for DIF is context effect. This means that the contextual scenarios (nonessential surface features) in which an item is situated lead to the differential functioning of an item [31]. Suppose that two groups of students have the same proficiency or mastery level of a tested concept. However, one group of students happens to be more familiar with the contextual features (for example, the wording, language, or storylines) of an item, but not necessarily what the item is intended to test. As a result, this group of students is likely to have a higher success rate on this item than those who are not familiar with the contextual features of the question. This context effect in essence speaks to item validity, which involves the issue of what exactly the item is testing
$[30,32]$. Oftentimes, a DIF caused by the context effect is deemed as undesirable [31].

\section{A. Potential DIF in the Force Concept Inventory}

As with many other assessments, the FCI is frequently used to compare students' Newtonian conceptions between different physics courses [32,33]. Anchoring in the above framework, it is logical to expect to see differential functioning in some FCI items if a comparison is performed between curricula that differ in their content. As mentioned above, there are two underlying causes that can lead to DIF: content and context. Typically, context effects are considered only after content effects are ruled out [34]. That is, only when students in different courses have similar access to the tested content will the attempt of seeking contextbased DIF be considered meaningful $[31,32]$. In the present study, the two curricula under investigation-M\&I and a PRTC calculus-based introductory mechanics coursediffer vastly in their goals and emphases of content. Therefore, detected DIF in the study represents a divergence in the learning opportunities that the courses provide to students.

In addition, the contextual features of the FCI items all involve everyday types of common objects and situations [20], such as moving aircrafts and colliding vehicles, familiar to both the students in M\&I and those in the traditional course. In fact, given that the FCI was intentionally designed to use only commonplace scenarios and natural language, it hardly makes a case to argue that students in any introductory mechanics course would be unfamiliar with the contexts of the FCI questions. Furthermore, in terms of the format of the FCI, students from both courses have experienced the multiple-choice question format used by the FCI in a number of previous courses. To this end, it is reasonable to attribute detected DIF in this study to content effects or the differences in course instruction.

\section{METHODOLOGY}

\section{A. Student participants and settings}

The data sample analyzed in this study is drawn from the work of Caballero et al. [16] conducted with science and engineering students taking calculus-based introductory mechanics at the Georgia Institute of Technology (Georgia Tech). This sample consists of 1013 pretest and post-test matched student data sets on the FCI, including 577 from a PRTC and 436 from the M\&I mechanics course. Although this sample is a subset of what was examined in the study of Caballero et al. (due to a crash of the original data storage system), it is representative of the reported sample in both overall scores and item scores (see Sec. V).

At the time the data were collected, M\&I was offered at Georgia Tech alongside a PRTC course that used 
Knight's [35] Physics for Scientists and Engineers as the text. Students in both the PRTC and M\&I mechanics courses attended three 1-hour large theater-style lectures ( 200 students) per week. Instruction in both lecture settings was pedagogically reformed, making use of "clicker questions" and Peer Instruction [4] with similar frequency.

In addition, students in both courses attended weekly labs and recitations. The M\&I students met in a single room for a 3-hour combined lab and recitation section each week. In the first hour, students completed recitation activities, typically consisting of solving conceptual problems in small groups. In the final two hours, students conducted laboratory activities including bench-top experiments and computational modeling. Graduate and undergraduate teaching assistants facilitated all the activities; the studentto-instructor ratio was typically 10:1. In the PRTC course, students met for a 1-hour recitation section and, separately, for a 2-hour laboratory. Recitation sections in the PRTC course made use of group problem-solving activities, often adopted from the University of Washington tutorials [8]. In their laboratory sections, students completed bench-top experiments, which were facilitated exclusively by graduate students. The student-to-instructor ratio in the recitation and the laboratory was 20:1.

As reported by Caballero et al., the FCI pretest was administered as a written test in the first week during either a lab session or a lecture to all the attending students in both courses. The post-test was also administered as a written test to the M\&I students in the last lab and to the PRTC students in an added evening test session. A small incentive accounting for $0.5 \%$ of student final grades was granted to those in the PRTC course (which was shown by Caballero et al. [16] to have a negligible effect on the results), whereas no incentives were offered to the M\&I students.

\section{B. Mantel-Haenszel method}

In this study, we use the Mantel-Haenszel (MH) [28] method to detect DIF in FCI questions. Item-responsetheory (IRT-)based techniques also can be used to identify DIF but require fitting empirical data to IRT models as a prerequisite $[19,28]$. We chose the $\mathrm{MH}$ method in our study because of its straightforward and robust nature as opposed to other methods [19]. More importantly, our initial analysis of the FCI data using the one-parameter IRT shows a poor fit to the model, and other IRT models involve two or more parameters, hence adding more complexity to inference making that hinges on the between-group comparisons. So, the MH method is a more viable choice than IRT-based approaches in this case.

The MH method is a stratum-specific approach to comparing student performance on individual items between two groups $[19,28]$. Students are stratified into
TABLE I. A cross-table showing student success and failure frequencies on an item at the $i$ th stratum measured by the FCI total scores in each course.

\begin{tabular}{lccc}
\hline \hline & Correct (1) & Incorrect (0) & Total \\
\hline M\&I & $a_{i}$ & $b_{i}$ & $a_{i}+b_{i}$ \\
PRTC & $c_{i}$ & $d_{i}$ & $c_{i}+d_{i}$ \\
Total & $a_{i}+c_{i}$ & $b_{i}+d_{i}$ & $N_{i}=a_{i}+b_{i}+c_{i}+d_{i}$ \\
\hline \hline
\end{tabular}

different levels (strata) based on their total test scores. In this study, the two groups of students are those enrolled in the M\&I mechanics course and their peers in a PRTC mechanics course. Students of each group are divided into 31 levels of proficiency based on their total FCI scores (which range from 0 to 30). At each stratum, a comparison between the two groups is carried out using an odds ratio (ratio of success odds), then a weighted average across all the strata is computed to identify potential DIF in each item. For example, Table I is a cross-table, illustrating the frequencies of students in each group who successfully and unsuccessfully answer a question at the $i$ th stratum. The odds ratio at this stratum is simply $a_{i} d_{i} / b_{i} c_{i}$ (the ratio of the "success versus failure" odds in the M\&I course to that in the PRTC course), which reveals whether student performance between the two groups is comparable at the $i$ th stratum. If the odds ratio value is equal or near 1, the two groups are considered to have a similar performance at this stratum. A weighted average across all the 31 strata then yields a full picture of the stratified comparison, which is often referred to as the Mantel-Haenszel alpha [28]:

$$
\alpha_{\mathrm{MH}}=\frac{\sum_{i} a_{i} d_{i} / N_{i}}{\sum_{i} b_{i} c_{i} / N_{i}}
$$

For each item, the MH alpha is tested for its significance using the chi-square distribution [28]. In this study, we use 0.01 as a significance threshold to control type I error (equivalent to a $99 \%$ confidence level). This is because, given the sample size in the present study, the likelihood of misidentifying DIF that in fact does not exist (false positive) is our major concern. Since it is desirable that we remain conservative in making claims about what we detect being true signals rather than artifacts, it is useful to raise the significance level. Additionally, using an error rate of 0.01 (or an even more stringent level) for $\mathrm{MH}$ test is a common practice in educational and medical sciences $[36,37]$.

For convenience, the $\mathrm{MH}$ alpha is often transformed into a logarithm function called MH D-DIF [28]

$$
\mathrm{MHD}-\mathrm{DIF}=-2.35 \ln \left(\frac{\sum_{i} a_{i} d_{i} / N_{i}}{\sum_{i} b_{i} c_{i} / N_{i}}\right) .
$$

Depending on the argument in the logarithm (greater or smaller than 1), the MH D-DIF can result in either a 
positive or a negative value. A positive value, as evident from the layout of Table I, indicates a DIF in favor of the PRTC course; or simply put, when matching on mastery levels measured by the FCI total scores, students in the PRTC course have higher odds of answering the question correctly than their peers in the M\&I course. Conversely, a negative value indicates a DIF in favor of the M\&I students. In addition, the magnitude of the MH D-DIF value shows the effect size of the between-group difference. According to the Educational Testing Services (ETS) [38], a DIF with MH D-DIF less than 1 in absolute value is deemed as negligible even if its significance output shows otherwise. Following this guideline, we flag items for potential DIF if the MH D-DIF is greater than 1 in absolute value [39] and its significance level is greater than 99\% $(p<0.01)$.

\section{DATA ANALYSIS AND FINDINGS}

\section{A. Conventional analysis}

To demonstrate that the sample under investigation is representative of the original larger sample in the study by Caballero et al., we first conduct conventional analyses to compare student performance in the M\&I and PRTC courses.

Table II shows the reliability indices (KR-20 reliability) for the pre- and post-FCI tests in both of the two courses. As seen, all of the values are above 0.80 , indicating a consistency in the test results sufficient for further analysis [40,41]. Although these results have not been previously reported in Caballero's work, we believe they form an important piece of evidence for establishing the validity of our subsequent investigation and inferences.

In our sample, the overall pretest scores for the PRTC course and the M\&I course are $46 \%(\mathrm{SD}=3 \%)$ and $45 \%$ $(\mathrm{SD}=4 \%)$, respectively, with no significant difference between them $(p>0.05)$. As for the post-test, the average scores for the PRTC and M\&I courses are $69 \%(\mathrm{SD}=3 \%)$ and $58 \%(\mathrm{SD}=6 \%)$, respectively, with a significant between-group difference at the level of $p<0.001$. In addition, it is found that students in the PRTC course on average achieved a normalized gain of $47 \%(\mathrm{SD}=2 \%)$, more than twice the average gain of $22 \%(\mathrm{SD}=5 \%)$ achieved by the M\&I students. These results are in close resemblance to those previously reported in Caballero's

TABLE II. Reliability indices (KR-20) for the FCI pretests and post-tests in both courses.

\begin{tabular}{lccccc}
\hline \hline & \multicolumn{2}{c}{ M\&I } & & \multicolumn{2}{c}{ PRTC } \\
\cline { 2 - 3 } \cline { 5 - 6 } \cline { 5 - 6 } & Pretest & Post-test & & Pretest & Post-test \\
\hline Reliability & 0.85 & 0.85 & & 0.85 & 0.83 \\
\hline \hline
\end{tabular}

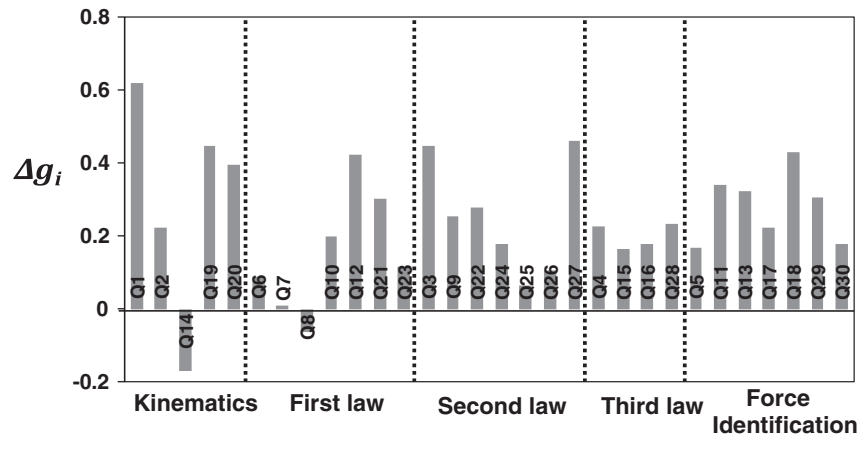

FIG. 2. Conventional analysis of differences in student performance on the individual FCI items between the PRTC course and the M\&I course: $\Delta g=g_{\text {PRTC }}-g_{\text {M\&I}}$. Here, each $g$ represents an item normalized gain.

work [pretest: $49 \%(\mathrm{SD}=2 \%)$ for PRTC course and $48 \%$ $(\mathrm{SD}=3 \%)$ for M\&I; posttest: $72 \%(\mathrm{SD}=2 \%)$ for PRTC course and $59 \%(\mathrm{SD}=3 \%)$ for $\mathrm{M} \& \mathrm{I}$; normalized gain: $46 \%(\mathrm{SD}=2 \%)$ for PRTC course and $23 \%(\mathrm{SD}=2 \%)$ for $\mathrm{M} \& \mathrm{I}]$.

On the individual items, we calculate, as was done in Caballero et al. [16], item normalized gains $g=$ $\left(f_{\text {post }}-f_{\text {pre }}\right) /\left(1-f_{\text {pre }}\right)$ for both the M\&I and PRTC courses. Here, $f_{\text {pre }}$ and $f_{\text {post }}$ represent the proportions of correct answers to an item on the pretest and post-test, respectively. A difference between the PRTC and M\&I courses is then calculated for each item $\Delta g=g_{\mathrm{PRTC}^{-}}$ $g_{\mathrm{M \& I}}$. The results are graphed in Fig. 2. As seen, the students in the PRTC course outperformed their M\&I peers on nearly all the items and topics covered by the FCI, again a finding consistent with those in the prior literature.

\section{B. DIF analysis of the FCI questions}

We use the Mantel-Haenszel method to detect DIF for both the pretest and post-test data. As mentioned before, we consider a DIF significant only if MH D-DIF is greater than 1 in absolute value and its significance rate is $p \leq 0.01$. Figure 3 shows the MH D-DIF values and their corresponding significance levels for the 1013 pretest and post-test matched data sets (see also the appendix). In the graphs, the number at the end of each bar is the significance level. Those with both $|\mathrm{MHD}-\mathrm{DIF}|>1$ and $p \leq 0.01$ are highlighted with color.

As evident from Fig. 3, no significant DIFs are detected for all the items in the pretest except for Q17, Q23, and Q27. Among them, Q17 is in favor of the students in the PRTC course, whereas Q23 and Q27 are in favor of the M\&I students. To a large extent, this result is intuitively expected, because to the best of our knowledge no systematic factors seemed to have influenced student choice of enrollment between the two courses, and students in both courses presumably should have had a similar mastery of the tested concepts before instruction. In addition, as pointed out by Caballero et al. [16], students' 


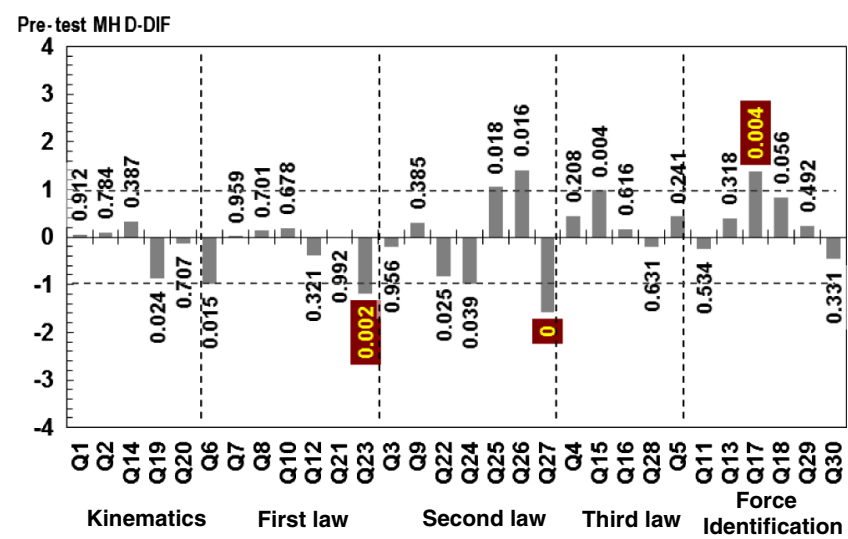

(a)

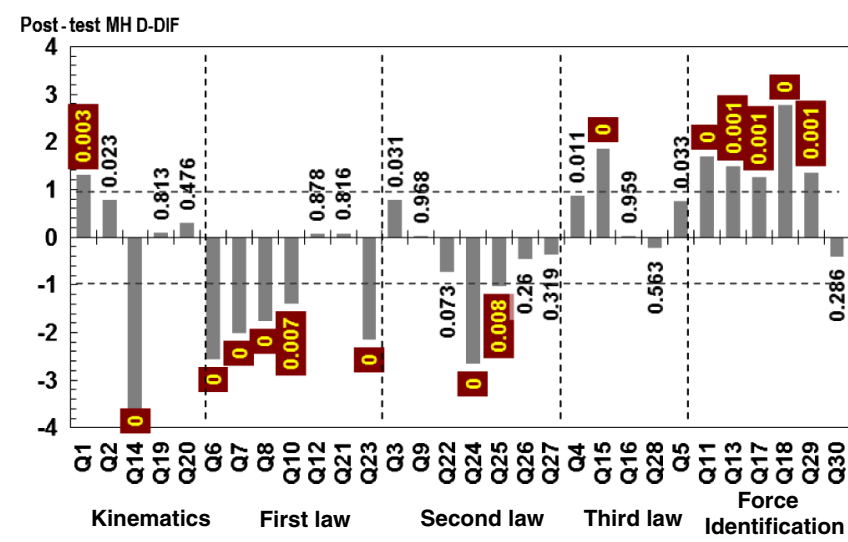

(b)

FIG. 3. MH D-DIF analysis. (a) MH D-DIF for pretest, (b) MH D-DIF for post-test. A positive MH D-DIF indicates an item in favor of the PRTC course; a negative MH D-DIF indicates an item in favor of the M\&I course. The numerical value on top of each bar shows the corresponding significance level ( $p$ value). Those with both $|\mathrm{MHD}-\mathrm{DIF}|>1$ and $p \leq 0.01$ are highlighted with color.

academic backgrounds as measured by their SAT scores and course grades in Calculus I showed no significant difference, further suggesting a similarity in student academic backgrounds between the two courses prior to instruction. However, the detected DIFs in the three items are puzzling, because no particular reasons can readily justify why students in one group would have outperformed the other before formal instruction. That said, given the fact that the large majority of the FCI items do not display significant DIFs in the pretest, we focus on these items for analysis and interpretation.

Contrary to the pretest results, the post-test analysis shows that there is a significant DIF in half of the FCI items, split between favoring the PRTC course and favoring the M\&I course. To better understand the patterns, we group all the items into five major topics targeted by the FCI as was done in the study by Caballero et al. (see Table III). Items showing a DIF in favor of the PRTC course
TABLE III. Categorization of the FCI items and detected DIFs (in bold prints). "+" indicate items in favor of the PRTC course, and " - " indicate items in favor of the M\&I course. " $*$ " indicate items with DIF in the pretest.

\begin{tabular}{lc}
\hline \hline Topics & $\begin{array}{c}\text { Items (“+”: favoring PRTC; “-”: } \\
\text { favoring M\&I) }\end{array}$ \\
\hline Kinematics & $\mathbf{1}+, 2, \mathbf{1 4}-, 19,20$ \\
Newton's first law & $\mathbf{6}-, \mathbf{7}-, \mathbf{8}-, \mathbf{1 0}-, 12,21, \mathbf{2 3}-*$ \\
Newton's second law & $3,9,22, \mathbf{2 4}-, \mathbf{2 5}-, 26,27 *$ \\
Newton's third law & $4, \mathbf{1 5}+, 16,28$ \\
Identification of forces & $5, \mathbf{1 1}+, \mathbf{1 3}+, \mathbf{1 7}+*, \mathbf{1 8}+, \mathbf{2 9}+, 30$ \\
\hline \hline
\end{tabular}

are marked with a "+" sign, and those of a DIF in favor of the M\&I course are marked with a "-" sign. By so doing, a new pattern starts to emerge; that is, the majority of the "+" items fall into the category of identification of forces and one item into Newton's third law, whereas most of the "--" items are in the categories of Newton's first and second laws. In addition, there is no strong evidence for advantage of either group on the kinematics topics. These findings, particularly the one regarding the M\&I students' performance on Newton's laws, are in conflict with the conclusions drawn in the prior studies, in which the M\&I students were found to underperform their peers in the PRTC course on nearly all the Newton's laws questions (cf. Fig. 2).

The above results can also be confirmed and further explicated by using item characteristic curves that display the proportion of correct answers at each mastery level measured by the FCI total scores. These curves readily reveal higher-order information on what the stratified differences look like as well as where such differences may reside-information that aggregate comparisons fail to reveal. In Fig. 4, two curves are depicted for each item with a DIF, representing the PRTC and M\&I courses, respectively. To increase the readability of the graphs while still maintaining their authenticity, we use a coarse size of 10 strata to represent 10 levels of mastery. In these figures, each circle marks the correct proportion of the M\&I students at every mastery level, while each triangle corresponds to the PRTC course. The curves are bestfitting logistic regression lines common for analysis of binary data (i.e., 1 for correct answers and 0 for incorrect answers, as is the case in our study). Here, the solid curves represent the M\&I course and dotted curves represent the PRTC course. As seen, there are betweengroup divergences, and more importantly, some of the divergences indeed reveal a better performance of the M\&I course when matching on student mastery levels. Also, these curves reveal the approximate locations where the between-group differences are mostly manifested. Some differences are fairly consistent across all the stratum (e.g., Q17), indicating that students at all 

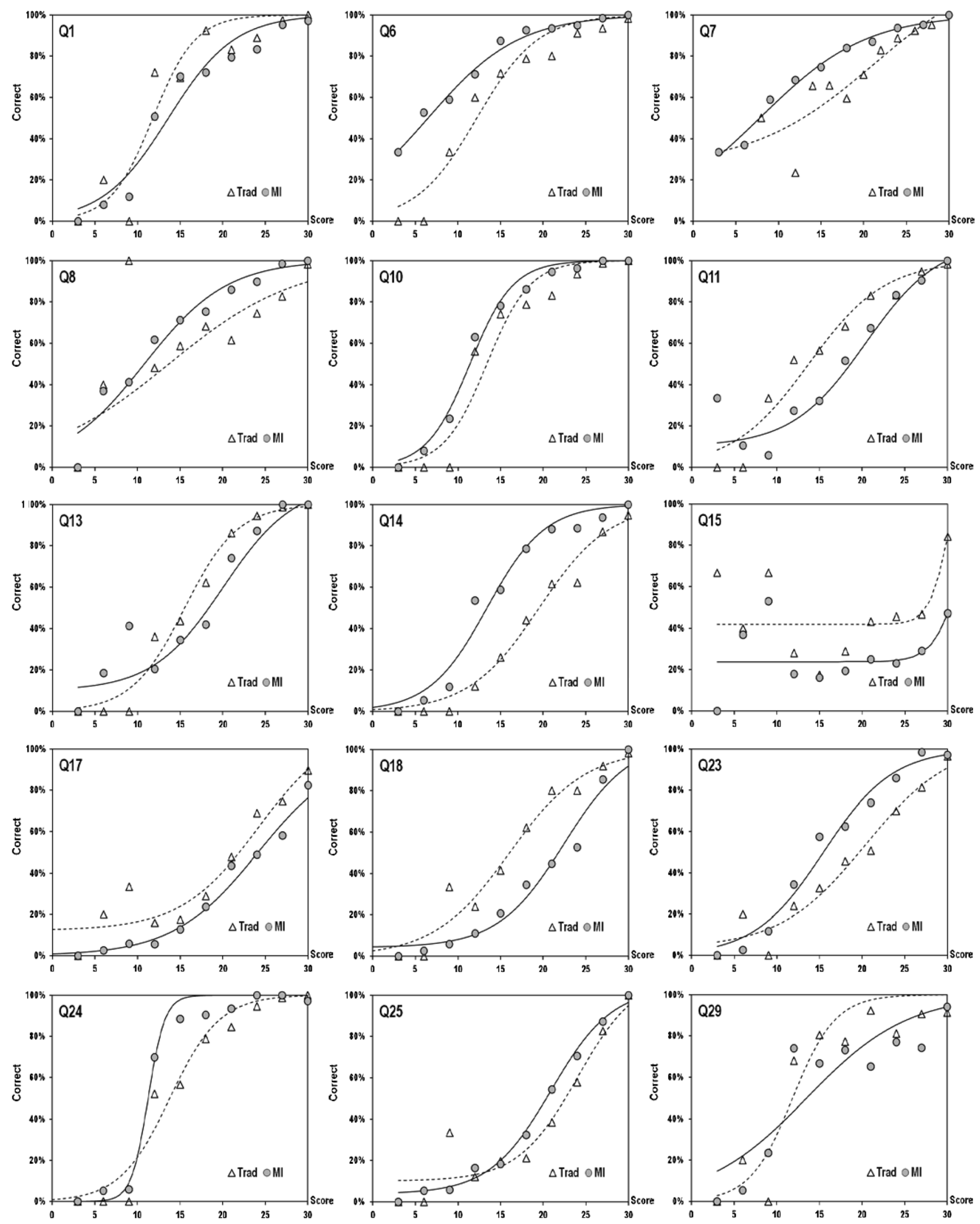

FIG. 4. Item characteristic curves for the FCI items with a significant DIF. The circular and triangular marks represent empirical observations of correct portions for the M\&I and PRTC courses, respectively. The solid and dashed curves are regression lines for the M\&I and PRTC courses, respectively. 
mastery levels in one course outperformed their matched counterparts in the other, whereas some differences are mainly located either at the lower end (e.g., Q6) or at the higher end (e.g., Q8) of the stratum, suggesting that it is primarily those with either high or low mastery levels that displayed a better performance in one course than their matched counterparts in the other. Additionally, some differences (although significant cumulatively across all the stratum levels as computed through the MH D-DIF) seem to reveal no clear visual pattern (e.g., Q13 and Q29), yielding relatively poor fit of the regression curves in a few cases (e.g., Q29). [Interestingly enough, in the case of Q15, while the between-group differences across all the strata are visibly clear, the regression fit is poor $\left(r^{2}=0.42\right.$ for the dashed line and $r^{2}=0.22$ for the solid line; see the appendix for a regression fit of other items with DIF). Nevertheless, our chief purpose of introducing the regression lines is not to seek any specific model fit, but to visually represent the stratum-specific differences between the two courses and to identify approximately at which level(s) such differences may reside.]

\section{DISCUSSION AND IMPLICATIONS}

The above results clearly indicate that the presence (or absence) of DIF in FCI items is influenced by whether the test is administered before or after instruction. The large majority of the items do not display significant DIFs in the pretest, whereas half of the items reveal significant DIFs in the post-test. It is worth reemphasizing that in this study we consider a DIF as significant only if its MH D-DIF is greater than 1 in absolute value and its significance level is at $p \leq 0.01$. This criterion allows us to filter out potential false positives and increases our confidence in the veridicality of the DIFs we have detected.

Since the DIFs predominantly occur in the post-test, it is reasonable to attribute their presence to the differing instruction students have received. As mentioned before, there are two underlying causes for DIF: content and context [31] (see Sec. III). Besides the aforementioned rationale for considering content effects in our study, the fact that only a few items display DIF in the pretest provides further empirical evidence for the minimal context effects, if any, of the FCI items on the differences in student performance.

Linking the detected DIFs to course instruction helps reveal some key differences between the two curricula under investigation (see Sec. III). It is evident from the results that the students in the PRTC course have a noticeably better performance on identification of forces and a slightly better performance on Newton's third law, a result that is generally consistent with those reported by
TABLE IV. Estimated fractions of homework questions and lecture devoted to the subtopics tested in the FCI. Reproduced from Caballero et al. [16] with permission.

\begin{tabular}{lrrrrr}
\hline \hline & \multicolumn{2}{c}{$\begin{array}{c}\text { Fraction of } \\
\text { homework questions }\end{array}$} & & \multicolumn{2}{c}{$\begin{array}{c}\text { Fraction of } \\
\text { lecture }\end{array}$} \\
\cline { 2 - 3 } \cline { 5 - 6 } & \multicolumn{1}{c}{ M\&I } & PRTC & & M\&I & PRTC \\
\hline Kinematics & 0.10 & 0.26 & & 0.07 & 0.21 \\
Newton's first law & $<0.01$ & $<0.01$ & & 0.02 & 0.01 \\
Newton's second law & 0.15 & 0.25 & & 0.09 & 0.08 \\
Newton's third law & $<0.01$ & 0.04 & & 0.01 & 0.03 \\
Force identification & 0.01 & 0.11 & & 0.06 & 0.11 \\
\hline \hline
\end{tabular}

Caballero et al. and is also justifiable in terms of student exposure to these topics. As discussed in the literature and shown in Table IV (reproduced from Ref. [16]), students in the PRTC course are frequently assigned homework problems and exposed to lecture and reading materials relating to force identification, more so than their peers in the M\&I course [16]. A similar case is for Newton's third law, in which students in the PRTC course receive a slightly higher fraction of lecture and homework on this topic [16].

On the other hand, our results also indicate that the M\&I students show an advantage on the majority of the items regarding Newton's first law and on a few items regarding Newton's second law (see Fig. 3 and Table III). Moreover, there is no strong evidence suggesting that M\&I students lag behind their PRTC course peers with the same level of mastery on the kinematics topics. These findings clearly are at odds with those derived from the conventional between-group comparisons as reported in the prior study [16].

In fact, to a large extent, the results emerging from our current study are sensible in view of students' exposure to the relevant topics in their respective courses. According to Caballero et al. (also see Table IV), the relative fraction of homework on Newton's first law is similar in both courses, but slightly more instruction is devoted to this topic in the M\&I class [16]. Perhaps more importantly, a few fundamental principles are consistently emphasized throughout the entire M\&I course. So, although the focal point of M\&I Modern Mechanics is not Newton's laws per se, the fact that the momentum principle $(\Delta \vec{p}=$ $\vec{F}_{\text {net }} \Delta t$ ) is introduced as a more generalizable form encompassing Newton laws has lent itself to a better appreciation of force-motion relations among the M\&I students [11]. As a result, their performance on the related items is likely to be positively influenced. The same line of reasoning can be applied to the case of Newton's second law, although the relative fractions of homework and lectures devoted to this topic are largely comparable in both courses [16]. 
As for the topic of kinematics, each of the two courses shows an advantage on one out of five relevant items, respectively (see Fig. 3 and Table III), and hence, no claims in favor of either course can be made. This finding in fact is not readily interpretable, particularly in light of the higher fractions of lecture and homework allocated to kinematics in the PRTC course. That said, alternatively, this result perhaps indicates that increased amount of instruction on kinematics in the PRTC course may only generate limited benefits for students' understanding of this topic, a claim worthy of further study.

Given the fact that the above findings are clearly incongruent with those from the conventional analysis reported in the prior study [16], a question worth asking is, Why do the same data yield different outcomes? The answer, in principle, lies in the different comparison methods. We argue that the conventional approach of comparing student performance on the individual items falls short of revealing additional information other than what has already been revealed in the total scores. In this case, the conventional result simply indicates that the students in the PRTC course demonstrated a better performance on the entire FCI than their M\&I peers, and that the same pattern is repeatedly observed across nearly all the individual items. Simply put, the group with a higher overall score also gets higher scores on the individual items. In fact, this is expected as long as the individual items are mutually consistent to form a valid assessment (as is the case for the FCI [42]). To that end, what we see from the comparisons on the individual items is no more than what we already know about the total score comparisons. Perhaps the only additional information we can extract from this result is that the individual FCI questions indeed are consistent with each other and hence can morph into a cohesive whole.

However, more information can be excavated when comparisons are made through matching on student mastery levels measured by the assessment in question. As such, the analysis is no longer aggregate but stratum specific; in other words, we compare apples with apples and not apples with oranges, so to speak, by putting students of the same mastery level side by side for matched comparisons. In fact, this is equivalent to controlling for student mastery levels and hence allows us to uncover some hidden information that is otherwise masked by the conventional approach [19].

Our study has clear implications for curriculum development and evaluation in general and for this work in particular. Our analysis speaks directly to the strengths and limitations of the M\&I mechanics course, providing evidence-based empirical accounts of the learning opportunities that students have on various topics of force and motion. In particular, our stratum-specific analysis suggests that instructors considering M\&I mechanics should recognize that students in their classes are likely to underperform on conceptual questions related to force identification and Newton's third law relative to students in PRTC courses. Efforts to develop instructional materials that help M\&I students build a stronger foundation in these areas are yet to be made. That said, there is a careful balance in altering M\&I mechanics to help students grasp concepts like those appearing on the FCI and losing the overarching narrative of the course. This, in fact, is a key feature that distinguishes M\&I mechanics from PRTC courses.

From the perspective of research methodology, our study exemplifies a finer-grained analysis approach that can lead to results in better alignment with actual classroom practices. Indeed, Caballero and colleagues were puzzled by their conventionally derived findings that students in the PRTC course outperformed their M\&I peers on Newton's first and second laws, while in fact the relative amount of instruction on these topics was comparable between the PRTC and M\&I courses with the latter being more principle oriented [16]. Our analysis and interpretation resolves this puzzle and clarifies some key differences between the two curricula. In a more general sense, what is presented in the study can be broadened out for various comparative educational studies. A range of issues, such as those regarding the divergence in curriculum impact on students of different socio-cultural backgrounds, genders, and ethnicities, can be better explored and explicated through fine-grained stratified comparisons $[37,43]$. Indeed, if used properly, the stratum-specific approach can provide a great deal of information about teaching and learning that will be appreciably useful for curriculum reform and improvement.

\section{ACKNOWLEDGMENTS}

The authors wish to thank Michael F. Schatz, Edwin F. Greco, and Eric R. Murray for helping to collect the data presented in this manuscript. The authors also thank Richard R. Hake and Katherine N. Mollohan for their insightful comments on the manuscript. 


\section{APPENDIX}

In what follows (Fig. 5), we report the numerical values of MH D-DIF for the individual FCI questions and their corresponding significance levels as shown in Fig. 3. Also presented herein (Table 5) is the logistic regression for each of the best fit curves displayed in Fig. 4.

\begin{tabular}{lcccc}
\hline & \multicolumn{2}{c}{ Pre-test } & \multicolumn{2}{c}{ Post-test } \\
\cline { 3 - 5 } Item & MH D-DIF & Significance $(p)$ & MH D-DIF & Significance $(p)$ \\
\hline Q1 & 0.052 & 0.912 & 1.300 & 0.003 \\
Q2 & 0.092 & 0.784 & 0.771 & 0.023 \\
Q3 & -0.212 & 0.956 & 0.773 & 0.031 \\
Q4 & 0.435 & 0.208 & 0.867 & 0.011 \\
Q5 & 0.432 & 0.241 & 0.761 & 0.033 \\
Q6 & -0.973 & 0.015 & -2.564 & 0.000 \\
Q7 & 0.019 & 0.959 & -2.019 & 0.000 \\
Q8 & 0.132 & 0.701 & -1.770 & 0.000 \\
Q9 & 0.301 & 0.385 & 0.014 & 0.968 \\
Q10 & 0.186 & 0.678 & -1.391 & 0.007 \\
Q11 & -0.251 & 0.534 & 1.706 & 0.000 \\
Q12 & -0.395 & 0.321 & 0.073 & 0.878 \\
Q13 & 0.395 & 0.318 & 1.481 & 0.001 \\
Q14 & 0.320 & 0.387 & -3.687 & 0.000 \\
Q15 & 0.978 & 0.004 & 1.861 & 0.000 \\
Q16 & 0.172 & 0.616 & 0.024 & 0.959 \\
Q17 & 1.368 & 0.004 & 1.257 & 0.001 \\
Q18 & 0.832 & 0.056 & 2.764 & 0.000 \\
Q19 & -0.867 & 0.024 & 0.096 & 0.813 \\
Q20 & -0.134 & 0.707 & 0.291 & 0.476 \\
Q21 & -0.005 & 0.992 & 0.082 & 0.816 \\
Q22 & -0.820 & 0.025 & -0.733 & 0.073 \\
Q23 & -1.182 & 0.002 & -2.155 & 0.000 \\
Q24 & -0.980 & 0.039 & -2.660 & 0.000 \\
Q25 & 1.050 & 0.018 & -1.034 & 0.008 \\
Q26 & 1.396 & 0.016 & -0.454 & 0.260 \\
Q27 & -1.582 & 0.000 & -0.362 & 0.319 \\
Q28 & -0.197 & 0.631 & -0.221 & 0.563 \\
Q29 & 0.237 & 0.492 & 1.363 & 0.001 \\
Q30 & -0.447 & 0.331 & -0.400 & 0.286 \\
\hline & & & &
\end{tabular}

TABLE V. Coefficient of determination $\left(r^{2}\right)$ for logistic regression curves in Fig. 4. Dashed curves represent the PRCT course, and solid curves represent the M\&I course. Note the regression curves are introduced solely for the purpose of visual representation and are not investigated for any particular model fit.

\begin{tabular}{lcc}
\hline \hline & \multicolumn{2}{c}{ Logistic regression $r^{2}$} \\
\cline { 2 - 3 } Item & $\begin{array}{c}\text { Dashed curve } \\
\text { (PRCT) }\end{array}$ & $\begin{array}{c}\text { Solid curve } \\
\text { (M\&I) }\end{array}$ \\
\hline Q1 & 0.89 & 0.95 \\
Q6 & 0.94 & 0.98 \\
Q7 & 0.80 & 0.98 \\
Q8 & 0.86 & 0.94 \\
Q10 & 0.94 & 0.98 \\
Q11 & 0.95 & 0.93 \\
Q13 & 0.98 & 0.92 \\
Q14 & 0.98 & 0.97 \\
Q15 & 0.41 & 0.22 \\
Q17 & 0.91 & 0.97 \\
Q18 & 0.96 & 0.97 \\
Q23 & 0.95 & 0.97 \\
Q24 & 0.95 & 0.98 \\
Q25 & 0.92 & 0.99 \\
Q29 & 0.86 & 0.78 \\
\hline \hline
\end{tabular}

FIG. 5. MH D-DIF values and significance levels for the FCI items. Items containing significant DIF are highlighted.

[1] W. Wood and J. Gentile, Teaching in a research context, Science 302, 1510 (2003).

[2] E. F. Redish, Teaching Physics with the Physics suite (John Wiley \& Sons Hoboken, NJ, 2003), p. 217.

[3] C. Wieman and K. Perkins, Transforming physics education, Phys. Today 58, No. 11, 36 (2005).
[4] E. Mazur, Peer Instruction: A User's Manual (PrenticeHall, Upper Saddle River, NJ, 1997), p. 253.

[5] L. Ding, N. W. Reay, A. Lee, and L. Bao, Exploring the role of conceptual scaffolding in solving synthesis problems, Phys. Rev. ST Phys. Educ. Res. 7, 020109 (2011). 
[6] E. Brewe, Modeling theory applied: Modeling instruction in introductory physics, Am. J. Phys. 76, 1155 (2008).

[7] J. Gaffney, E. Richards, M. B. Kustusch, L. Ding, and R. Beichner, Scaling up education reform, J. Coll. Sci. Teach. 37, 48 (2008).

[8] L. C. McDermott and P. S. Shaffer, Tutorials in Introductory Physics (Prentice Hall Upper Saddle River, NJ, 2002).

[9] E.-S. Morote and D. Pritchard, What course elements correlate with improvement on tests in introductory Newtonian mechanics?, Am. J. Phys. 77, 746 (2009).

[10] R. Beichner, R. Chabay, and B. Sherwood, Labs for the Matter \& Interactions curriculum, Am. J. Phys. 78, 456 (2010).

[11] R. Chabay and B. Sherwood, Modern mechanics, Am. J. Phys. 72, 439 (2004).

[12] R. Chabay and B. Sherwood, Restructuring the introductory electricity and magnetism course, Am. J. Phys. 74, 329 (2006).

[13] R. Chabay and B. Sherwood, Computational physics in the introductory calculus-based course, Am. J. Phys. 76, 307 (2008).

[14] L. Ding and X. Liu, in Getting Started in PER-Reviews in $P E R$, edited by $\mathrm{C}$. Henderson and K. Harper (American Association of Physics Teachers College Park, MD, 2012), Vol. 2, pp. 1-42.

[15] M. Kohlmyer, M. Caballero, R. Catrambone, R. Chabay, L. Ding, M. Haugan, J. Marr, B. Sherwood, and M. Schatz, Tale of two curricula: The performance of 2000 students in introductory electromagnetism, Phys. Rev. ST Phys. Educ. Res. 5, 020105 (2009).

[16] M. Caballero, E. Greco, E. Murray, K. Bujak, M. Marr, R. Catrambone, M. Kohlmyer, and M. Schatz, Comparing large lecture mechanics curricula using the Force Concept Inventory: A five thousand student study, Am. J. Phys. 80, 638 (2012).

[17] E. H. Haertel and W. A. Lorie, Validating standards-based test score interpretations, Measurement 2, 61 (2004).

[18] R. J. Mislevy, L. S. Steinberg, and R. G. Almond, On the structure of educational assessments. Measurement 1, 3 (2003).

[19] B. Clauser and K. Mazor, Using statistical procedures to identify differential functioning test items, Educ. Meas. 17, 31 (1998).

[20] D. Hestenes, M. Wells, and G. Swackhamer, Force Concept Inventory, Phys. Teach. 30, 141 (1992).

[21] R. Chabay and B. Sherwood, Matter \& Interactions I: Modern Mechanics, 3rd ed. (John Wiley \& Sons Inc., New York, 2011).

[22] L. Ding, R. Chabay, and B. Sherwood, How do students in an innovative principle-based mechanics course understand energy concepts?, J. Res. Sci. Teach. 50, 722 (2013).

[23] R. Chabay and B. Sherwood, Bringing atoms into first-year physics, Am. J. Phys. 67, 1045 (1999).

[24] A. Cromer, Stable solutions using the Euler approximation, Am. J. Phys. 49, 455 (1981).

[25] D. Scherer, P. Dubois, and B. Sherwood, VPython: 3D interactive scientific graphics for students, Comput. Sci. Eng. 2, 56 (2000).

[26] M. Caballero, M. Kohlmyer, and M. Schatz, Implementing and assessing computational modeling in introductory mechanics, Phys. Rev. ST Phys. Educ. Res. 8, 020106 (2012).

[27] R. L. Linn, in Differential Item Functioning, edited by P. W. Holland and H. Wainer (Lawrence Erlbaum Associates Hillsdale, NJ, 1993), pp. 349-364.

[28] S. J. Osterlind and H. T. Everson, Differential Item Functioning, 2nd ed. (Sage Publications Thousand Oaks, CA, 2009).

[29] N. Raju and B. Ellis, in Measuring and Analyzing Behavior in Organizations: Advances in Measurement and Data Analysis, edited by F. Drasgow and N. Schmitt (JosseyBass San Francisco, 2002), pp. 156-188.

[30] G. Camilli, in Educational Assessment, edited by R. L. Brennan (Praeger Publishers, West Port, CT, 2006), pp. 221-256.

[31] M. Michaelides, A review of the effects on IRT item parameter estimates with a focus on misbehaving common items in item equating, Front. Psychol. 1, 1 (2010).

[32] L. Ding, Seeking missing pieces in science concept assessments: Reevaluating the Brief Electricity and Magnetism Assessment through Rasch analysis, Phys. Rev. ST Phys. Educ. Res. 10, 010105 (2014).

[33] R. R. Hake, Interactive-engagement versus traditional methods: A six-thousand-student survey of mechanics test data for introductory physics courses, Am. J. Phys. 66, 64 (1998).

[34] G. Camilli, in Differential Item Functioning, edited by P. Holland and H. Wainer (Lawrence Erlbaum Associates Hillsdale, NJ 1993), pp. 387-413.

[35] R. Knight, Physics for Scientists and Engineers (AddisonWesley, San Francisco, 2008).

[36] B. Clauser, K. Mazor, and R. Hambleton, The effects of purification of matching criterion on the identification of DIF using the Mantel-Haenszel procedure, Appl. Meas. Educ. 6, 269 (1993).

[37] R. Dietz, R. Pearson, M. Semark, and C. Willis, Gender bias in the Force ConceptInventory?, AIP Conf. Proc. 1413, 171 (2012).

[38] R. Zwick, A Review of ETS Differential Item Functioning Assessment Procedures: Flagging Rules, Minimum Sample Size Requirements, and Criterion Refinement (Educational Testing Service Princeton, NJ, 2012).

[39] ETS considers $|\mathrm{MHD}-\mathrm{DIF}|>1.5$ as an indication of large between-group differences. However, its use as a cutoff criterion for significance has been reported to be too strict and often leads to over-diagnosis of DIF. See Ref. [38].

[40] L. Ding and R. Beichner, Approaches to data analysis of multiple-choice questions, Phys. Rev. ST Phys. Educ. Res. 5, 020103 (2009).

[41] L. Ding, R. Chabay, B. Sherwood, and R. Beichner, Evaluating an electricity and magnetism assessment tool: Brief electricity and magnetism assessment, Phys. Rev. ST Phys. Educ. Res. 2, 010105 (2006).

[42] T. F. Scott and D. Schumayer, Exploratory factor analysis of a Force Concept Inventory data set, Phys. Rev. ST Phys. Educ. Res. 8, 020105 (2012).

[43] E. Davidov, P. Schmidt, and J. Billiet, Cross-Cultural Analysis: Methods and Applications (Routledge, New York, NY, 2011). 